\title{
Detailed optical behavior of the 2000 outburst of the eclipsing recurrent nova $\mathrm{CI}$ Aquilae
}

\author{
K. Matsumoto ${ }^{1, \star}$, M. Uemura ${ }^{1}$, T. Kato ${ }^{1}$, S. Kiyota ${ }^{2}$, K. Ayani ${ }^{3}$, T. Kawabata ${ }^{3}$, L. Král ${ }^{4}$, T. Havlík ${ }^{4}$, \\ M. Kolasa ${ }^{4}$, R. Novák ${ }^{5}$, and G. Masi ${ }^{6}$ \\ 1 Department of Astronomy, Graduate School of Science, Kyoto University, Kyoto 606-8502, Japan \\ 2 Center for Balcony Astrophysics, 1-401-810 Azuma, Tsukuba 305-0031, Japan \\ 3 Bisei Astronomical Observatory, 1723-70 Ohkura, Bisei, Okayama 714-1411, Japan \\ 4 Observatory and Planetarium of Johann Palisa, 17. listopadu 15, 70833 Ostrava, Czech Republic \\ 5 Nicholas Copernicus Observatory, Kraví hora 2, Brno 616 00, Czech Republic \\ ${ }^{6}$ Center for Backyard Astrophysics Italy, Via Madonna de Loco, 47, 03023 Ceccano, Italy
}

Received 21 May 2001 / Accepted 6 August 2001

\begin{abstract}
The 2000 outburst of the recurrent nova CI Aql was followed by optical photometry and spectroscopy. Our time-resolved photometry revealed its intraday variations during the outburst. The orbital modulation of the light curve appeared after entering the plateau stage. We found that primary eclipses were $\sim 0.6$ mag in depth, but the profile of the eclipse was significantly different from that in the quiescent phase. The folded orbital light curve was represented by a wide wing of a primary eclipse and it suggests the existence of the accretion disk at the plateau stage. In this outburst, we obtained accurate determination of several minima of primary eclipses, and found that the timings of minima showed a substantial delay compared to the previously reported ephemeris. However, no significant evidence of a change in the orbital period was observed since the discovery of the eclipsing nature of this object. We examined the evolution of optical spectra through the outburst, which reconfirmed the nova nature of this object. A spectrum taken on 2000 October 10 showed the $\mathrm{H} \alpha$ in emission and indicated that the object had not yet reached quiescence. The overall light curve and late-stage spectroscopy have revealed that the plateau is the longest one among recurrent novae.
\end{abstract}

Key words. accretion: accretion disks - stars: binaries: eclipsing - stars: individual: CI Aql - stars: novae, cataclysmic variables

\section{Introduction}

Recurrent novae are a subclass of cataclysmic variables (CVs; see Warner 1995 for a review). While the classification is simply based on observational records reporting more than two times of nova explosion, the significant differences between recurrent novae and classical novae are smaller amplitudes and higher frequency of outbursts of the former, i.e., all novae are believed to be essentially recurrent. It is widely accepted that the white dwarfs in recurrent novae are very massive and close to the Chandrasekhar limit, and the mass-transfer rate from the companion is relatively high, namely an order of $\sim 10^{-7} M_{\odot} \mathrm{yr}^{-1}$. The existence of massive white dwarfs and the high mass-transfer rates in recurrent novae bring causes of the differences in the observational

Send offprint requests to: K. Matsumoto,

e-mail: katsura@cc.okayama-u.ac.jp

* Present address: Graduate School of Natural Science and Technology, Okayama University, Tsushima-naka 3-1-1, Okayama 700-8530, Japan. properties, such as the frequent explosions with 10-100 yr intervals and the smaller released energies in outbursts $\left(10^{43}-10^{44} \mathrm{erg}\right)$ compared to those of classical novae. Before the second detection of a nova explosion of CI Aql in 2000, eight such recurrent systems had been known.

The optical light curve of CI Aql in quiescence indicates that this object is an eclipsing binary with an orbital period of 0.618355 d (Mennickent \& Honeycutt 1995, hereafter referred as MH1995). The sinusoidal orbital modulation, with an amplitude of $0.2 \mathrm{mag}$, is seen at out of the primary eclipse showing 0.6 mag depth.

The optical spectral feature is much hard to interpret. Its spectrum taken in quiescence shows weak Balmer lines in absorption on the redder continuum $(B-V \sim$ 1.0) in addition to absorption lines of $\mathrm{NaD}$, K I $\lambda 7696$, Ca II $\lambda \lambda 8542,8662$, and TiO bands (Greiner et al. 1996). These Balmer and metallic absorption lines generally suggest a non-CV nature for this object. On the contrary, the lines of HeII $\lambda 4686$ and CIII-N III complex at $\sim 4640 \AA$ 
are in emission, along with the above absorption lines (Greiner et al. 1996). This object was transiently detected by ROSAT PSPC (Greiner et al. 1996).

The historical detection of an outburst was made in June 1917 (Reinmuth 1925). Due to the lack of more detailed information for the 1917 event, the nature of this object had remained unsettled. Duerbeck (1987) suggested the classification for a possible nova or dwarf nova, based on the low amplitude of the outburst.

In 2000 April, a "new star" at the location of CI Aql was independently detected by two amateur astronomers, Kesao Takamizawa and Minoru Yamamoto, on their patrol films taken on 2000 April 28.668 (UT) and 28.694 (UT), respectively, as a $\sim 10.0 \mathrm{mag}$ object (Takamizawa et al. 2000). Takamizawa noted that there was no additional detection on his plates of this field between 1994 May 12 and 2000 April 11 (Takamizawa et al. 2000), and Liller (2000) also reported that the object was not brighter than $\sim 11$ mag on his orange-red photographs taken on between 1983 October and 2000 April 4.333 (UT). The original suspected-CV classification had almost been disregarded due to the spectroscopic features at that time (e.g., Downes et al. 1997).

Following these observations, immediate spectroscopic exposures for the probable nova were made on 2000 April 29.6 (UT) by using a 1.88-m telescope at the Okayama Astrophysical Observatory of National Astronomical Observatory, Japan (Uemura \& Kato 2000). The spectra clearly showed a strong $\mathrm{H} \alpha$ emission line with FWHM $2300 \mathrm{~km} \mathrm{~s}^{-1}$ and confirmed that it was a nova outburst in slightly evolved stage. The remaining problem had been whether the outbursting object was exactly CIAql, the possible Nova Aql 1917, or not. It was solved by detailed astrometry of the object, which showed that the coordinate is in good agreement with that of CI Aql (Yamaoka et al. 2000). Thus, it was confirmed that CI Aql underwent the second-recorded explosion in 2000, 83 years after the previous one in 1917, and that it is the ninth recurrent nova we have known at present.

In this paper, we report our optical photometric and spectroscopic observations of the 2000 outburst of CI Aql. We followed this event photometrically from immediately after the discovery, with intraday time-resolved samplings, and succeeded to detect some minima of eclipses in the light curves. Optical spectra of the object were taken at four epochs in 2000, including the first snapshot by Uemura \& Kato (2000), which derived an evolution of the spectral features during this outburst.

\section{Observations}

\subsection{Photometry}

The optical photometric observations were carried out on 140 nights between 2000 April 29 and 2001 April 30 at the following six sites: Tsukuba, Ibaraki, Japan $\left(140^{\circ} 5^{\prime} \mathrm{E}\right.$; "Tsukuba" in Table 1), the Ouda station, Department of Astronomy, Kyoto University, Japan (13557' E; "Ouda" in Table 1), Department of Astronomy, Kyoto University, Japan (135 47' E; "Kyoto" in Table 1), Observatory and Planetarium of Johann Palisa, VSB-Technical University Ostrava, Czech Republic (18 $9^{\prime}$ E; "Ostrava1" in Table 1), Nicholas Copernicus Observatory, Brno, Czech Republic $\left(16^{\circ} 35^{\prime} \mathrm{E}\right.$; "Brno" in Table 1), and Ceccano, Italy $\left(13^{\circ} 20^{\prime} \mathrm{E}\right.$; "Ceccano" in Table 1$)$.

The data reductions and analyses for each observation were performed by following methods: for the Tsukuba observation, the MIRA $A P^{1}$ was used; for the Ouda observation, the IRAF software package ${ }^{2}$ and tasks in the apphot aperture photometry package were mainly used; for the Kyoto observations, a Java ${ }^{\mathrm{TM}}$-based aperture and PSF photometry package which was developed by one of the authors (Kato) was used. We adopted aperture photometry for the data prior to 2000 November 19 and then PSF photometry for the following data due to the faintness of the object; for the Brno observation, the Munipack ${ }^{3}$ was used; for the Ostrava and Ceccano observations, the Munidos ${ }^{4}$ was used.

The brightness of the object was determined by relative photometry. The comparison stars were GSC 5114.149 for the Ouda, Kyoto1, and Kyoto2 runs and GSC 5114.651 for the Tsukuba run. Their constancies were checked with GSC 5114.584. For the Ostrava observations, GSC 5114.651 and GSC 5114.273 were respectively used as comparison and check stars in the May and June runs (Ostrava1), and GSC 5114.149 and USNO 0825.13285694 were respectively used as them in the July run (Ostrava2). For the Brno observation, GSC 5114.540 and HD 17647 were used as comparison stars, and we confirmed the constancy within $0.03 \mathrm{mag}$, by comparison with some nearby bright stars. Information on reference stars for the Ceccano observation was unfortunately lost due to an accident in its archive. For the observations except the Ceccano run, the photometric sequence of this field by Henden (2000) was used.

\subsection{Spectroscopy}

The optical spectroscopic observations were carried out at the Bisei Astronomical Observatory, Japan $\left(133^{\circ} 33^{\prime} \mathrm{E}\right)$ on three nights of 2000 May 20, July 9 (Bisei1), and October 10 (Bisei2), by using a 1.01-m telescope with a

\footnotetext{
1 MIRA AP is a software platform for calibrating and processing in astronomy produced by Axiom Research, USA. See http://www.axres.com/MIRA-AP.html for more information.

${ }^{2}$ IRAF is distributed by the National Optical Astronomy Observatories, which are operated by the Association of Universities for Research in Astronomy, Inc., under cooperative agreement with the National Science Foundation. See http://iraf .noao.edu/ for more information.

${ }^{3}$ Munipack is a stellar photometry package developed by Filip Hroch. The core of the photometry is based on the DAOPHOTII by Peter B. Stetson in the ESO MIDAS. See http://munipack.astronomy.cz/ for more information.

4 Munidos is an implementation on DOS of the Munipack, which was made by two of the authors (Novák \& Král).
} 
Table 1. A summary of particular information concerning the photometric observations for each observatory (see Sect. 2.1). Note that the filter used in the Ostrava runs was R of the RGB system, whose transmission characteristics can be found at http://www.sbig.com/sbwhtmls/Oldfilterchart.htm

\begin{tabular}{lllllc}
\hline Obs. code & Reflector and optical system & \multicolumn{1}{c}{ Detector } & Filters & Observing period & Nights \\
\hline Tsukuba & 0.25-m Schmidt-Cassegrain & SITe SIA502AB & $V, R_{\mathrm{c}}, I_{\mathrm{c}}$ & 2000 May 4-2001 Feb. 16 & 31 \\
Ouda & 0.60 -m Ritchey-Chretien & SITe SI004AB & $R_{\mathrm{c}}$ & 2001 Mar. 9 & 1 \\
Kyoto1 & 0.25 -m Schmidt-Cassegrain & Kodak KAF-0400 & none & 2000 May 1-2001 Apr. 30 & 105 \\
Kyoto2 & 0.25 -m Schmidt-Cassegrain & Kodak KAF-0401E & $R_{\mathrm{c}}$, none & 2000 Oct. 30-2000 Dec. 15 & 27 \\
Ostrava1 & 55-mm photo-lens & Kodak KAF-0400 & $R$ & 2000 May 6-2000 Jun. 2 & 5 \\
Ostrava2 & 0.15 -m Coude & Kodak KAF-0400 & $R$ & 2000 Jul. 5 & 1 \\
Brno & $0.4-\mathrm{m}$ Newtonian & Kodak KAF-0400 & $V, R_{\mathrm{c}}$ & 2000 Apr. 29-2000 May 9 & 4 \\
Ceccano & $0.28-\mathrm{m}$ Schmidt-Cassegrain & Kodak KAF-0400 & none & 2000 Jun. 20 & 1 \\
\hline
\end{tabular}

classical Cassegrain. We used two detectors of a liquidnitrogen cooled CCD (EEV CCD15-11/UV) for Bisei1 and an electronically cooled CCD (Kodak KAF-1600) for Bisei2. The dispersion was $162 \AA / \mathrm{mm}$ corresponding to $\sim 4.5 \AA /$ pixel image scale. The wavelength range was $4400 \AA$ (centered at $5500 \AA$ ) for Bisei1 and $2300 \AA$ (centered at $7000 \AA$ ) for Bisei2. The integration time was set to 1200 s. Due to the sensitivity of the CCD, a marginal part on the blue side of each frame was chopped prior to the reducing procedure for the Bisei1 observation. Data reductions and analyses were processed with tasks in the onedspec package in the IRAF, for the Bisei1, and with an interactive processing software for data reductions of spectral CCD data, developed by one of the authors (Kawabata), for the Bisei2. Fe-Ne comparison lamp was used for wavelength calibrations, and HR 7596 (=58 Aql) was used as a standard star for flux calibrations which were applied for the Bisei1 only.

\section{The 2000 outburst of $\mathrm{Cl} \mathrm{Aql}$}

\subsection{Overview}

In Fig. 1, the overall $R_{\mathrm{c}}$ light curve of the 2000 outburst of CI Aql is depicted. The light curve indicates that the object was approximately $8 \mathrm{mag}$ around the maximum and thus the amplitude of the outburst was likely about 6 mag. The brightness turned to decline on May $8-9$, and the major decline continued till June 14 . Then, the object entered a long plateau stage.

In the early part of the outburst, the object kept a constant magnitude between May 2 and 8 except for a small variability with apparent amplitude of $\sim 0.2$ mag. This variability seems to be independent of the orbital modulation, according to the magnitudes on May 4 and 5 , and no intraday variation was seen at this stage.

The major declining trend was observed accompanying strange rebrightening (or large fluctuation), and the amplitude of the hump was at least $\sim 1$ mag. The magnitudes on May 14 and 15 suggest an appearance of plural humps during this declining phase, and the VSNET data $\operatorname{archive}^{5}$ (e.g., referred in Kiss et al. 2001) also supports a few rebrightenings. The nightly light curves during this declining stage were dominated by small-scale but clear variations which were still not related with any regularity like an orbital modulation. Near the end of this stage, however, gradually developing orbital modulations were apparently observed in the nightly light curves. The profile was not stable and not so clear due to contaminations by the small-scale variations superimposed on the orbital modulation.

The declining trend stopped at mid-June, and then a plateau stage started. According to the light curve, the plateau stage can be divided into three parts. The "first" part when the magnitude was almost constant was terminated by a $\sim 0.5$ mag drop of the light at between July 13 and 16. After the drop, the "second" part continued during about 160 days with a gradual declining trend, and the light curve leveled off, when the object was 1-2 mag brighter than in the quiescence.

The stability, however, was broken at around 2000 November 23 by a significant dip, i.e., the object suddenly faded to be about $15.5 \mathrm{mag}$ in $R_{\mathrm{c}}$. After the dip, the object immediately recovered within the following several days, and it seemed to be again stabilized at around 14 mag in $R_{\mathrm{c}}$, which was about 1 mag fainter than before the dip. Thus, the "third" part started after the dip, and it continued over the conjunction beginning in 2000 December. The continuation was also confirmed by the VSNET observations. Such a long duration of more than 300 days of the plateau phase is quite uncommon among outbursts of recurrent novae.

\subsection{Orbital modulation}

The orbital modulation clearly developed in the plateau stage. We performed a period analysis by a Phase Dispersion Minimization method (PDM; Stellingwerf 1978) for the light curves of the first and second plateau stages. Each nightly observation with a small number of data-points or large scatter was excluded prior to the

\footnotetext{
5 http://www.kusastro.kyoto-u.ac.jp/vsnet/gcvs/ AQLCI.html
} 


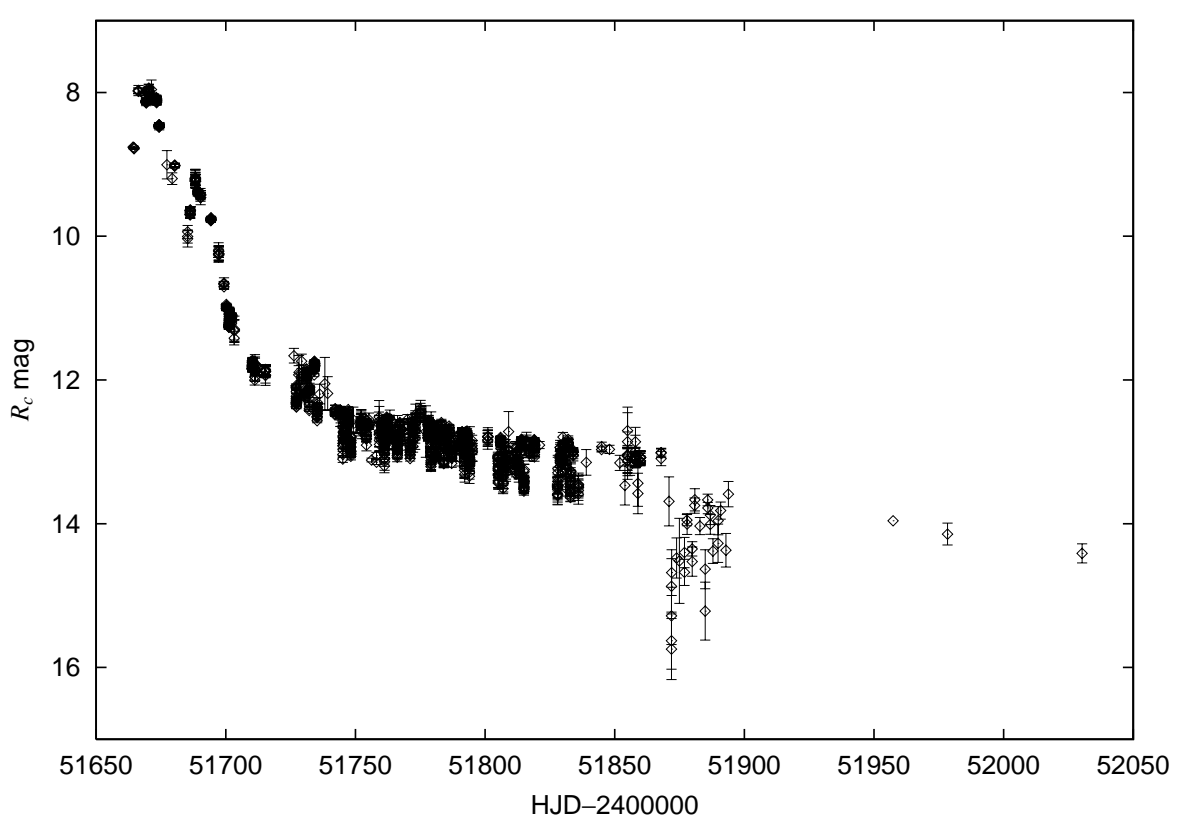

Fig. 1. The light curve of the 2000 outburst of CI Aql, consisting of Tsukuba, Ouda, Kyoto, Ostrava, and Brno data. The vertical and horizontal axes represent $R_{\mathrm{C}}$-magnitude and HJD-2 400000 , respectively. Data on individual nights were binned for clarity of the outline (the bins contain 20 data each except low-quality observations). Vertical scatters seen in each datum during the plateau stage were mainly caused by the orbital modulation in each fractional light curve.

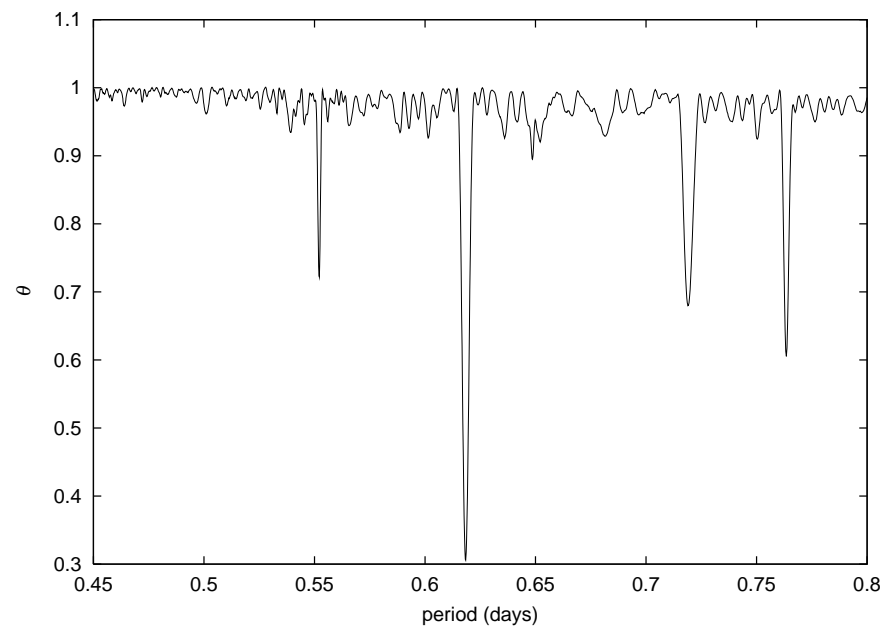

Fig. 2. A $\theta$-period diagram for the light curve during the second plateau stage.

analysis, and the two stages were individually processed. For the first plateau stage, no declining correction was applied. In the second plateau stage, the light curve changed its declining trends, and we therefore divided the light curve further into three sub-segments: July 16-September 17 (decline), September 18-October 21 (constant), and October 27-November 19 (decline). The declining trends were assumed to be linear, and were separately estimated and applied for the individual sub-segments. Then, the fragmental nightly light curves corresponding to boundaries of the sub-segments were adjusted relative to each other. The independent PDM analyses for the two plateau stages led a consistent result of a modulating period of $0.61835 \mathrm{~d}$ (Fig. 2), and this periodicity was consistent with the orbital period determined by MH1995 in the quiescence. The aliases of the best estimation in Fig. 2 were safely rejected by the primary minimum on HJD 2451731.5 and the next one, respectively observed in Ostrava2 and Kyoto1, i.e., folded light curves by the likely periods clearly distinguished the aliases.

Our time-resolved photometry successfully covered many primary minima of the orbital modulation, which made timings of the minima determinable for us. In the timings, we found a systematic delay of 0.01-0.02 d relative to expected ones based on the ephemeris given in MH1995 (Fig. 3). The O-C of the timings was determined by the Kwee \& van Woerden's method (Kwee \& van Woerden 1956), and we found that the $\mathrm{O}-\mathrm{C}$ is linear and constant with an average value of $+0.014 \pm 0.004$. The error was mainly caused by the short-time variability superposed on the orbital light curve, which affected to profiles of the primary minima and sometimes made the determinations confusing. The $\mathrm{O}-\mathrm{C}$ indicated a systematic difference of the zero point compared with the timing given in MH1995. We derived an updated ephemeris of the primary eclipses as,

$T_{\min }($ HJD $)=2451701.2086(89)+0.61835(10) \times E$.

The delay is within the error of the initial ephemeris in MH1995. Thus, it is concluded that we could not detect any evidence for significant change of the orbital period during the previous quiescent state, in contrast with the case of a recurrent nova USco in the 1999 outburst (Matsumoto et al. 2001).

Figure 4 shows the folded orbital light curve during the plateau stage. We can see that the full amplitude of the primary minimum was $\sim 0.6 \mathrm{mag}$, which indicates that 


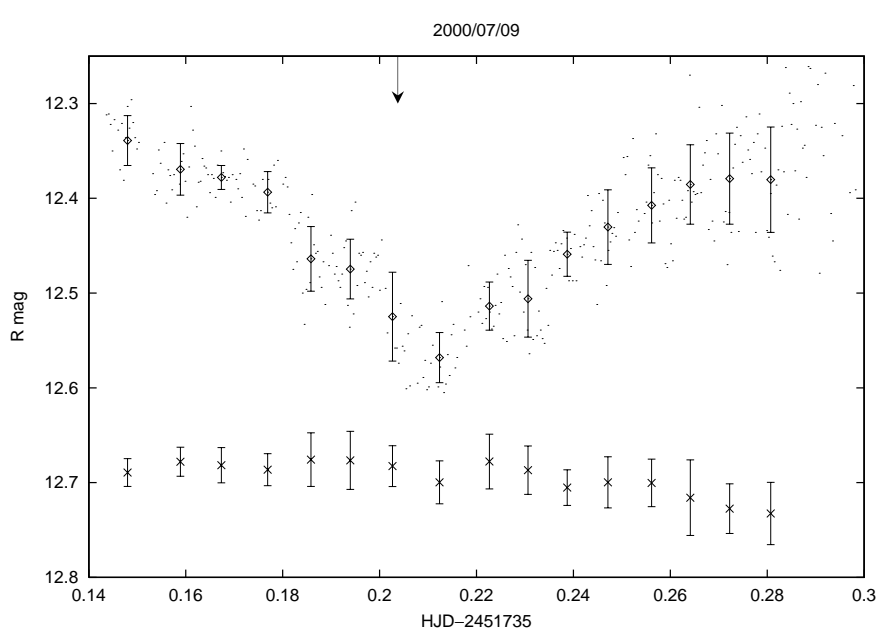

Fig. 3. An example of a primary eclipse developed in the plateau stage (2000 July 9). The diamond marks with error bars represent the magnitude of the object. The cross marks denote constancy of the comparison star throughout the same observing period, which are adjusted by an arbitrary constant for displaying. The vertical downward-arrow indicates the expected timing of the minimum, based on the ephemeris by MH1995. The exposure time was $30 \mathrm{~s}$, and the corresponding time-resolution was about $40 \mathrm{~s}$.

the orbital modulation completely appeared at that stage of the outburst. The primary eclipse had a very broad wing extended to $\sim 0.7$ orbital-phase duration, and the shape was generally stable throughout the plateau stage. The vertical scatters of the light curve were mainly caused by the short time variability, but it is noted that a disturbance was seen at orbital phase $\Phi_{\text {orb }}$ of $\sim 0.7-0.8$ in the light curve. Some nightly light curves covering this phase indeed showed such significant fluctuations.

\subsection{Spectral evolution}

We obtained optical spectra of the object at four epochs in this outburst, and pursued the evolution of the spectral features.

The top panel of Fig. 5 shows an early spectrum taken by Uemura \& Kato (2000) one night after the discovery, which gave the confirmation of the nova classification. Uemura \& Kato (2000) reported that this spectrum suggested characteristics of a fast nova at a few weeks after a maximum and that P Cygni profile was not seen in the Balmer lines. We were able to identify the strongest $\mathrm{H} \alpha$ and $\mathrm{H} \beta$ lines and emission lines of N II, FeII, and HeI. A relatively strong emission feature of N III-He II complex around $4650 \AA$ was also dominant, which likely consists of N III $\lambda 4640$ and He II $\lambda 4686$ emission components seen in quiescence. The general feature of the early spectrum was also seen in spectra taken on May 20 in the decline stage, but the domination of the $\mathrm{H} \alpha$ emission line developed more (middle panel of Fig. 5). The emission line at $\sim 5020 \AA$ is likely a complex of N II $\lambda 5001+$ He I $\lambda 5016+$ Fe II $\lambda 5018$ lines. In addition to this feature, the presence of the emission lines of the N III-He II and N II at $\sim 5700 \AA$

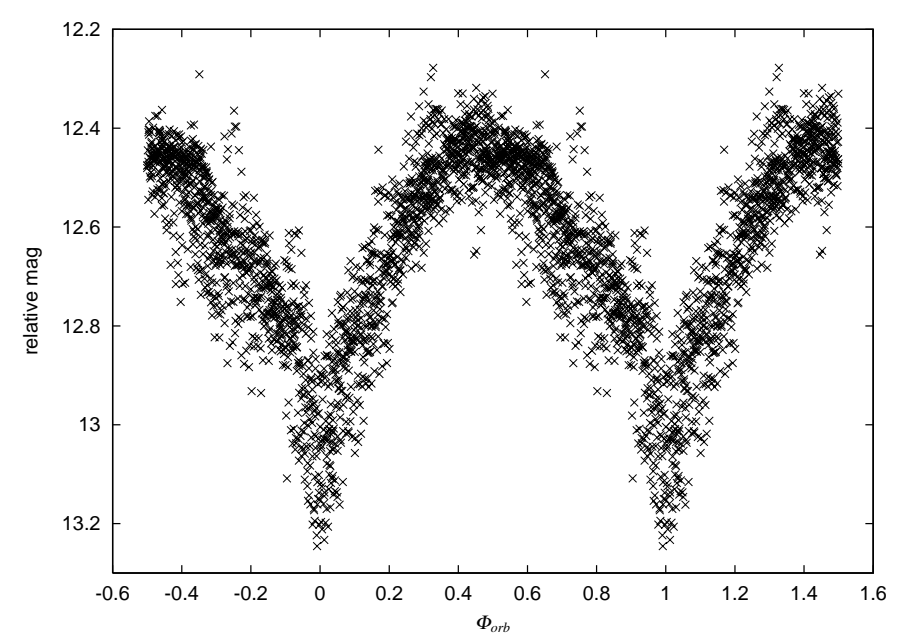

Fig. 4. The orbital light curve of CI Aql during the plateau stage. The new timing of minima (this paper) and the orbital period given in MH1995 were used. The bins contain 10 data points each for the $30 \mathrm{~s}$ exposures and the phase is repeated for clarity.

represents a characteristics of $\mathrm{He} / \mathrm{N}$-type novae spectra (Williams 1992), which was pointed out for this object by Kiss et al. (2001) based on their optical spectroscopic investigations for the relatively early phase of this outburst.

During the early plateau stage, the $\mathrm{H} \alpha$ emission line was still prominent, but optical spectral features significantly changed. [O III] multiple emission around $5000 \AA$ is easily identified and the intensity of N III-He II complex was relatively enhanced in the optical spectrum taken on 2000 July 9 (bottom panel of Fig. 5). The nebular spectrum indicates the existence of an extremely thin gas, which is consistent with the appearance of the orbital modulation in the light curve after starting the plateau stage. It is noted that the appearance of the nebular phase significantly contrasts with the absence of that in the optical spectrum of the recurrent nova U Sco in its outbursts. This suggests that the ejected mass in this event was larger than that in cases of USco, which is consistent with the estimates by Hachisu et al. (2000a) and Hachisu \& Kato (2001a).

The optical spectral evolution from a principal to a nebular spectrum was consistent with that typically seen in outbursts of classical novae, and thus reconfirmed that this outburst was indeed a nova explosion.

The spectra presented above were taken near primary minima, when the mass-donor star eclipsed the main component and the brightest part of the accretion disk. Thus the apparent absence of absorption features, likely caused from the secondary star, in the optical spectra may be remarkable. But it remains that low resolution could be the cause; in our low-resolution spectra, diffuse interstellar bands described in Kiss et al. (2001) were not resolved, and thus their $E(B-V)=0.85$ was applied to our dereddening. 

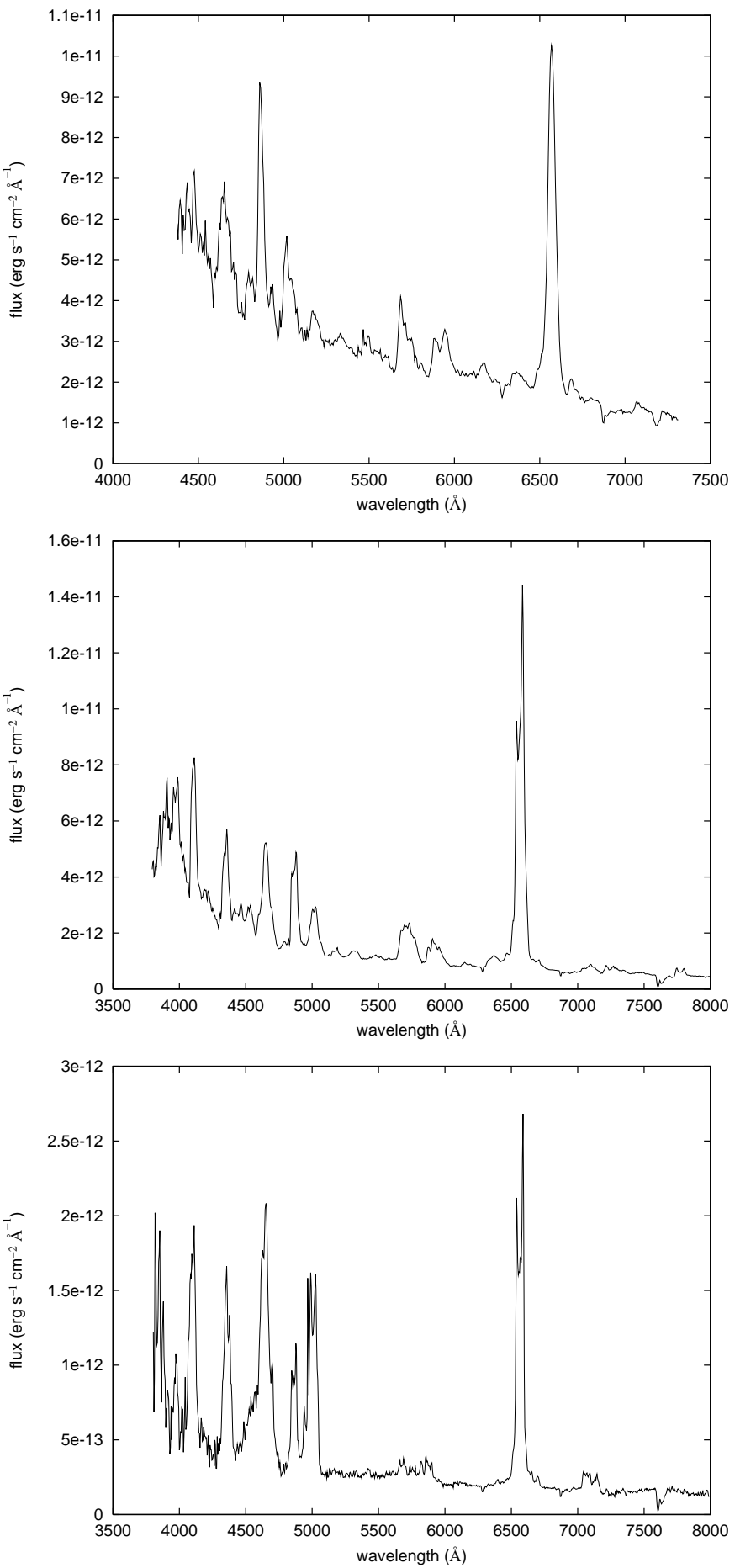

Fig. 5. [Top]: the optical spectrum of CI Aql taken on 2000 April 29.6 (UT), corresponding to the orbital phase of 0.08 (from the work of Uemura \& Kato 2000). [Middle]: the averaged spectrum taken on 2000 May 20.7 (UT), corresponding to the orbital phase of around 0.2. [Bottom]: the averaged spectrum taken on 2000 July 9.7 (UT), corresponding to the orbital phase of 0.43. The exposure time for Bisei data was $1200 \mathrm{~s}$. These spectra are displayed with dereddening of $E(B-V)=0.85$ (Kiss et al. 2001).

On 2000 October 10, a part of the long plateau stage, we also obtained an optical spectrum of the object, and the spectrum showed that the $\mathrm{H} \alpha$ was still significantly in

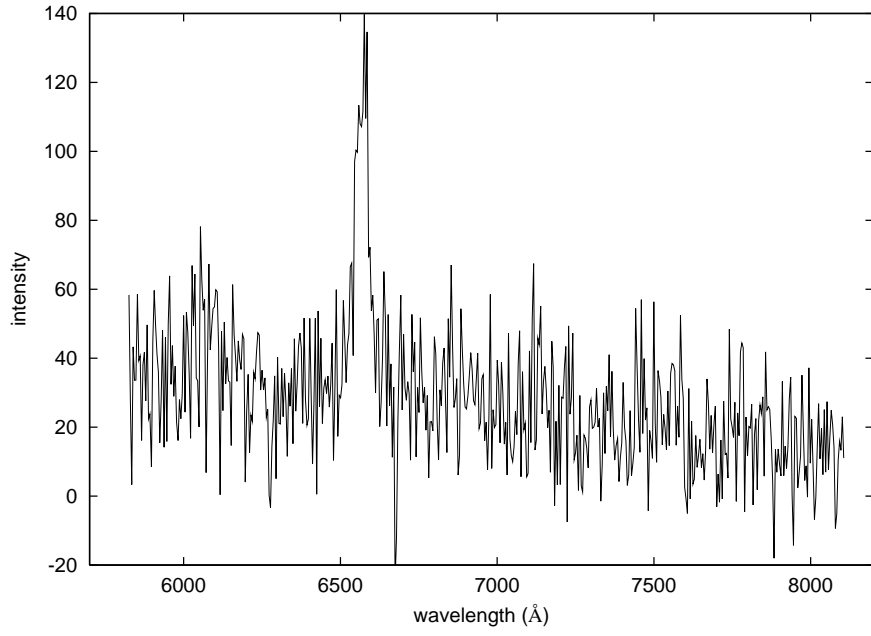

Fig. 6. The optical spectrum in the red region taken on 2000 October 10.47 (UT), corresponding to the orbital phase of 0.03 . The integration time was $300 \mathrm{~s}$.

emission (Fig. 6). Because the Balmer series should turn to absorption features in quiescence, we can expect that the existence of Balmer emission lines is a status indicator which indicates whether the object is in quiescence, and thus it was confirmed that the object was not in quiescence at least on that occasion.

\section{Discussions}

The overall light curve of this outburst indicated that CI Aql is a recurrent nova being a fast or moderately fast nova with a longer plateau prior to the end of outburst. The optical spectra and their evolution throughout this outburst confirmed its nova nature and the long duration of the plateau stage.

The apparent lower amplitude is a characteristic of this recurrent nova. However, it should be noted that the true maximum of this outburst might have happened earlier and was brighter than suggested by the presented data. Williams (2000) examined archival plates of the Harvard College Observatory and extracted the historical event in 1917. His result calibrated with Tycho- $B$ magnitude presented a peak and following decline in the 1917 outburst. The peak magnitude in the Tycho- $B$ was roughly equal to that of the 2000 outburst in $R_{\mathrm{c}}$. This indicates that the latter should be intrinsically fainter with regard to the reddening. Unfortunately, no observations are available for the object between 2000 April 11 and 27. Thus, a possible brighter and earlier maximum cannot be completely excluded.

Color indices derived from the data of Kyoto $\left(R_{\mathrm{c}}\right)$ and Tsukuba $(V)$ indicated that the object was getting bluer during the early part accompanying the major decline, and the transition into the plateau phase at mid-June coincides with the stopping of further progression toward the blue in $V-R$ (Fig. 7). Schmeja et al. (2000) made $I J K$-band photometry on that occasion, and the near-infrared color 


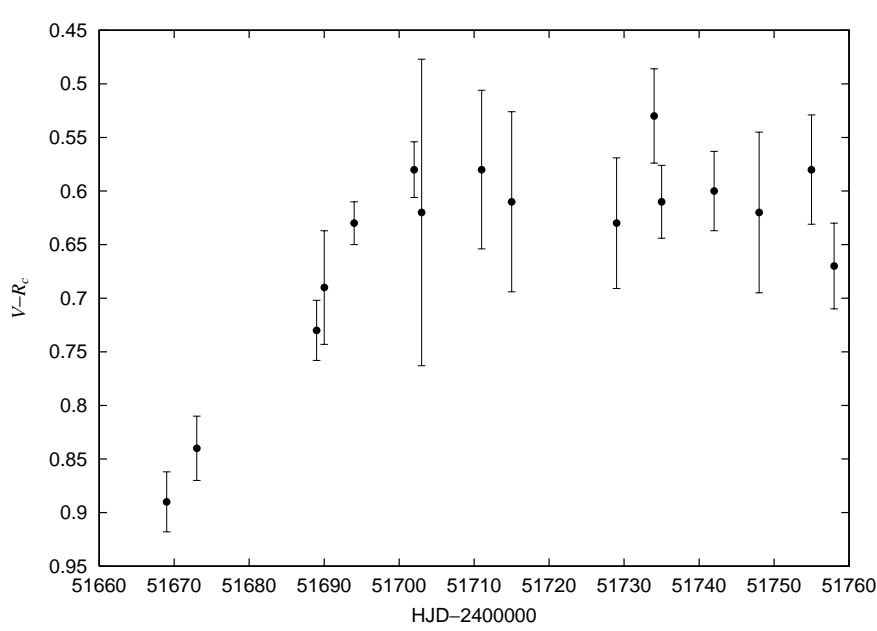

Fig. 7. The color change of the object $\left(V-R_{\mathrm{c}}\right)$ during the early part of the 2000 outburst.

also showed that both of the light and color curves were flattened around JD 2451720.

The orbital light curve of the object, appearing in the plateau stage, showed the clear primary minima with wide wings and no (or inconspicuous) secondary minima. The profile was stable except for the vertical scatters, and the secondary minima might have rather variable profiles. Wilson \& Dunscombe (2000) reported a self-absorption feature in a line at He I $1.0830 \mu \mathrm{m}$ on their infra-red spectra of this object, which suggests a relatively high inclination angle for the accreting binary system. At the same time, the primary minima indicated no totality in our result, even if they come from eclipses of the white dwarf by the mass-donor star. In addition, no absorption feature is visible in the optical spectra, although they were recorded near $\Phi_{\text {orb }} \sim 0$. Such observational aspects remind us of those of supersoft X-ray sources (SSSs). The relation between SSSs and recurrent novae is a current topic for discussion after TPyx (Patterson et al. 1998) and USco (Kahabka et al. 1999), with the possibility of being progenitor systems of type Ia supernovae (Hachisu et al. 1999; Hachisu \& Kato 2001b). The recurrent nova U Sco was detected as a transient SSS during the plateau stage in the 1999 outburst. The numerical model for the binary system of U Sco by Hachisu et al. (2000a, 2000b) showed that both the observed nova-explosion and the orbital light curve can be consistently reproduced by the model with considerations of the SSS-like configuration. They concluded that the white dwarf in U Sco should be massive and is a very probable candidate for a progenitor system of a type Ia supernova.

With the similarities described above, this work also revealed that CIAql has a slightly different nature to that of USco or usual SSSs in some aspects. In this outburst, the timescale of the transition was relatively long $\left(t_{3} \sim 35 \mathrm{~d}\right.$ if the maximum appeared on 2000 May 1$)$, and the amplitude was probably lower than those of other recurrent novae. Contrary to the case of U Sco, no significant change of the orbital period during the previous quiescence was detected.

The spectral features in this outburst showed that CIAql is obviously a CV, but this makes the optical spectrum in quiescence highly conspicuous. From this viewpoint, the co-existence of absorption features of the Balmer lines and emission ones of He II $\lambda 4686$ is similar to that of SMC 13 (Crampton et al. 1997) and RX J0537.7-7034 (Greiner et al. 2000). These two sources are classified as transient SSSs (Kahabka et al. 1994; Orio et al. 1997) and show such a symbiosis of absorption and emission lines (van Teeseling et al. 1998; Greiner et al. 2000). In this context, the model concerning a mild hydrogen flash for the short-period SMC 13-type SSSs (Kahabka \& Ergma 1997) may be also able to interpret the probable low amplitude of the outburst of this object, in addition to both the spectral feature of CI Aql during the quiescence and its possible relationship with SSSs. Here, a problem is the significant difference of the orbital periods between those systems and CI Aql.

It should be mentioned that there is a prediction that CI Aql is indeed in a "supersoft" phase during the third plateau stage of this outburst, according to light curve analyses based on an SSS-like configuration for the binary system of the U Sco-type recurrent novae (Hachisu \& Kato 2001a). If the drop in November was caused by a wind stop of the outburst, supersoft X-ray emission is expected to be emitted as a consequence of the hydrogen burning on the surface of the white dwarf. A significant point is that the theoretical model can consistently reproduce both the light curves of the outbursts and the orbital light curve during the quiescence of the object with a $\sim 1.2 M_{\odot}$ white dwarf. Thus, we conclude that we found the second likely example of a recurrent nova being in connection with the SSS-phenomenon.

Acknowledgements. The authors would like to express sincere gratitude to the VSNET members for their extensive monitoring, and to the referee László L. Kiss for his valuable comments and suggestions. The authors would also like to thank Ryuko Hirata for his improvements of the manuscript. K.M. and M.U. was financially supported by the Research Fellowships of the Japan Society for the Promotion of Science for Young Scientists.

\section{References}

Crampton, D., Hutchings, J. B., Cowley, A. P., \& Schmidtke, P. C. 1997 , ApJ, 489, 903

Downes, R. A., Webbink, R. F., \& Shara, M. M. 1997, PASP, 109,345

Duerbeck, H. W. 1987, Space Sci. Rev., 45, 1

Greiner, J., Alcala, J. M., \& Wenzel, W. 1996, IBVS, 4338

Greiner, J., Orio, M., \& Schwarz, R. 2000, A\&A, 355, 1041

Hachisu, I., \& Kato, M. 2001a, ApJ, 553, L161

Hachisu, I., \& Kato, M. 2001b, ApJ, accepted [astro-ph/0104040]

Hachisu, I., Kato, M., Kato, T., \& Matsumoto, K. 2000a, ApJ, 528, L97

Hachisu, I., Kato, M., Kato, T., et al. 2000b, ApJ, 534, L189 
Hachisu, I., Kato, M., \& Nomoto, K. 1999, ApJ, 522, 487

Henden, A. 2000, ftp://ftp.nofs.navy.mil/pub/outgoing/ aah/sequence/ciaql. dat

Kahabka, P., \& Ergma, E. 1997, A\&A, 318, 108

Kahabka, P., Hartmann, H. W., Parmar, A. N., \& Negueruela, I. 1999, A\&A, 347, L43

Kahabka, P., Pietsch, W., \& Hasinger, G. 1994, A\&A, 288, 583

Kiss, L. L., Thomson, J. R., Ogloza, W., et al. 2001, A\&A, 366,858

Kwee, K. K., \& van Woerden, H. 1956, Bulletin of the Astronomical Institutes of The Netherlands, 12, 327

Liller, W. 2000, IAUC, 7417

Matsumoto, K., Kato, T., \& Hachisu, I. 2001, PASJ, submitted

Mennickent, R. E., \& Honeycutt, R. K. 1995, IBVS, 4232 (MH1995)

Orio, M., Della Valle, M., Massone, G., \& Ögelman, H. 1997, A\&A, 325, L1
Patterson, J., Kemp, J., Shambrook, A., et al. 1998, PASP, 110,380

Reinmuth, K. 1925, Astron. Nach., 225, 385

Schmeja, S., Armsdorfer, B., \& Kimeswenger, S. 2000, IBVS, 4957

Stellingwerf, R. F. 1978, ApJ, 224, 953

Takamizawa, K., Kato, T., Yamamoto, M., et al. 2000, IAUC, 7409

Uemura, M., \& Kato, T. 2000, IAUC, 7409

van Teeseling, A., Reinsch, K., Pakull, M. W., \& Beuermann, K. 1998, A\&A, 338, 947

Warner, B. 1995, Cataclysmic Variable Stars (Cambridge University Press, Cambridge)

Williams, D. B. 2000, IBVS, 4904

Williams, R. E. 1992, AJ, 104, 725

Wilson, J. C., \& Dunscombe, K. R. 2000, IAUC, 7426

Yamaoka, H., Ayani, K., \& Shirakami, K. 2000, IAUC, 7411 